\title{
Perfil de crianças e adolescentes em cuidados paliativos no centro de terapia intensiva pediátrica oncológica
}

Profile of children and adolescents in palliative care at the pediatric oncology intensive care center Perfil de niños y adolescentes en cuidados paliativos en el centro de cuidados intensivos de oncología pediátrica

Amanda Danielle Resende Silva e Sousa ${ }^{1,2}$ (D) https://orcid.org/0000-0002-0450 -1967

Liliane Faria da Silva ${ }^{1}$ (D) https://orcid.org/0000-0002-9125-1053

Ana Carla Dantas Cavalcanti ${ }^{1}$ (D) https://orcid.org/0000-0003-3531-4694

Marcelle Miranda da Silva ${ }^{1}$ (D) https://orcid.org/0000-0003-4872-7252

Fernanda Garcia Bezerra Góes ${ }^{1}$ (D) https://orcid.org/0000-0003-3894-3998

Juliana Rezende Montenegro Medeiros Moraes ${ }^{1}$ (D) https://orcid.org/0000-0002-2234-6964

Cristineide dos Anjos ${ }^{1}$ (D) https://orcid.org/0000-0002-4614-3088

\section{Resumo}

Objetivo: Caracterizar o perfil clínico e demográfico de crianças e adolescentes internados no Centro de Terapia Intensiva Pediátrica Oncológica em cuidados paliativos exclusivos entre os anos de 2008 e 2018.

Métodos: Estudo retrospectivo, quantitativo, com análise de todos os prontuários de crianças e adolescentes em cuidados paliativos exclusivos $(n=57)$.

Resultados: Identificou-se que a maioria das internações (64,9\%) eram crianças, 85,9\% das crianças/ adolescentes residiam no Rio de Janeiro e $98,2 \%$ permaneceram acompanhados por familiares durante o período de internação. No quesito religião, $76,3 \%$ eram cristãos. Entre os tipos de neoplasia, o neuroblastoma foi 0 mais prevalente $(26,3 \%)$, e $93 \%$ dessas crianças/adolescentes foram a óbito no período investigado.

Conclusão: É importante conhecer o perfil clínico e demográfico dessas crianças/adolescente, com impossibilidade de tratamento modificador da doença, para que possam ser oferecidos cuidados paliativos de forma precoce, bem como valorizar as redes de apoio, valores, desejos e biografias de cada paciente e família.

\section{Abstract}

Objective: To characterize the clinical and demographic profile of children and adolescents admitted to the Pediatric Oncology Intensive Care Center in exclusive palliative care between 2008 and 2018.

Methods: Retrospective and quantitative study with analysis of all medical records of children and adolescents in exclusive palliative care $(n=57)$.

Results: We identified that most hospitalizations (64.9\%) involved children, $85.9 \%$ of children/adolescents lived in Rio de Janeiro and $98.2 \%$ remained accompanied by relatives during the hospitalization period. In terms of religion, $76.3 \%$ were Christians. Among the types of neoplasia, neuroblastoma was the most prevalent $(26.3 \%)$, and $93 \%$ of these children/adolescents died in the investigated period.

Conclusion: It is important to know the clinical and demographic profile of these children/adolescents, with the impossibility of treatment to modify the illness, so that palliative care can be offered early, besides enhancing the support networks, values, desires and biographies of each patient and family.

\section{Resumen}

Objetivo: Caracterizar el perfil clínico y demográfico de niños y adolescentes ingresados en el Centro de Cuidados Intensivos de Oncología Pediátrica en cuidados paliativos exclusivos entre los años 2008 y 2018.

Métodos: Estudio cuantitativo y retrospectivo con análisis de todos los registros médicos de niños y adolescentes en cuidados paliativos exclusivos $(n=57)$.

Resultados: Se identificó que la mayoría de las hospitalizaciones (64,9\%) abarcaba niños, el 85,9\% de los niños/ adolescentes residían en Río de Janeiro y el 98,2\% permanecían acompañados por parientes durante el período de hospitalización. En términos de religión, el 76,3\% eran cristianos. Entre los tipos de neoplasia, el neuroblastoma fue el más frecuente (26,3\%), y el $93 \%$ de estos niños/adolescentes murieron en el período investigado.

Conclusión: Es importante conocer el perfil clínico y demográfico de estos niños/adolescentes, con la imposibilidad de tratamiento para modificar la enfermedad, de modo que los cuidados paliativos puedan ofrecerse tempranamente, así como mejorarse las redes de apoyo, valores, deseos y biografías de cada paciente y familia.
Descritores

Neoplasias; Cuidados Paliativos; Unidade de Terapia Intensiva Pediátrica

\section{Keywords}

Neoplasms; Palliative Care; Pediatric Intensive Care Unit

\section{Descriptores}

Neoplasias; Cuidados Paliativos; Unidad de Cuidados Intensivos Pediátricos

\section{Como citar:}

Silva e Sousa AD, Silva LF, Cavalcanti AC, Silva MM, Góes FG, Moraes JR, et al. Perfil de crianças e adolescentes em cuidados paliativos no centro de terapia intensiva pediátrica oncológica. Rev Soc Bras Enferm Ped. 2020;20(2):80-6.

${ }^{1}$ Instituto Nacional de Câncer, Brasília, DF, Brasil.

${ }^{2}$ Universidade Federal Fluminense, Niterói, RJ, Brasil.

Conflitos de interesse: nada a declarar.

Autor correspondente: Amanda Danielle Resende Silva e Sousa | E-mail: amandadani@icloud.com

Submetido: 23 de Janeiro de 2020 | Aceito: 26 de Março de 2021

DOI: http://dx.doi.org/10.31508/1676-3793202000012 


\section{Introdução}

O câncer é a doença crônica que apresenta maior mortalidade na faixa etária de 1 a 19 anos no Brasil.(1) O Instituto Nacional de Câncer (INCA) estimou 12.500 novos casos de câncer em crianças e adolescentes para o biênio 2018-2019.(2)

Diante do adoecimento por câncer, a criança e o adolescente são retirados da sua vida social e se veem em uma situação em que a expectativa de futuro é duvidosa e a possibilidade de cultivar amizades e de ser feliz limitada.

As identificações do sofrimento, portanto, devem receber intervenções eficazes da equipe de saúde, de modo simultâneo ao tratamento dos sintomas físicos, para que os aspectos biopsicossocial, cultural e espiritual das crianças e adolescentes sejam manejados com a mesma eficácia. ${ }^{(3)}$

Esses cuidados que dão suporte aos aspectos biopsicossociais e espirituais das crianças, adolescentes e suas famílias são os cuidados paliativos (CP), definidos pela Organização Mundial da Saúde (OMS) como aqueles que melhoram a qualidade de vida dos pacientes e seus familiares que enfrentam problemas inerentes a uma doença que ameaça a vida. ${ }^{(4)}$

O CP é transversal ao tratamento, ou seja, ocorre do diagnóstico à cura ou fim de vida, por isso deve estar inserido em todos os cenários nos quais as crianças e os adolescentes se encontrem..$^{(3)}$ Contudo, são considerados exclusivos quando oferecidos às pessoas que não têm mais chances de cura da sua doença de base, assim não possuem plano terapêutico curativo, sendo este o único tratamento oferecido a partir desse momento. ${ }^{(3)}$

Levando-se em consideração que o câncer infantojuvenil é considerado um problema de saúde pública com dados epidemiológicos que revelam o aumento gradativo dessa patologia em todas as faixas etárias ${ }^{(2)}$ e que parte dos acometidos pode necessitar de cuidados paliativos exclusivos (CPE), torna-se necessário o desenvolvimento de estudos que caracterizem o perfil sociodemográfico, religioso e físico dessas crianças/adolescentes e suas famílias para que os profissionais e gestores das unidades de saúde possam organizar a oferta dos serviços de $\mathrm{CP}$ direcionados à sua clientela específica.

O objetivo deste estudo é caracterizar o perfil clínico e demográfico de crianças e adolescentes internados no Centro de Terapia Intensiva Pediátrica Oncológica em cuidados paliativos exclusivos entre os anos de 2008 e 2018.

\section{Métodos}

Estudo retrospectivo, de natureza quantitativa, no qual foram analisados os prontuários de crianças e adolescentes em CPE internados no Centro de Terapia Intensiva Pediátrica Oncológica (CTIPO) do Hospital do Câncer I, localizado na cidade do Rio de Janeiro, entre os anos de 2008 e 2018.

A pesquisa foi aprovada pelo Comitê de Ética da Universidade Federal Fluminense (UFF) e do Instituto Nacional do Câncer (INCA) com os seguintes números do parecer, respectivamente: 2.958 .989 e 2.989 .914 .

Para seleção da amostra do estudo, fez-se, inicialmente, uma busca por todas as internações de crianças e adolescentes que estiveram no CTIPO entre 1 de janeiro de 2008 e 31 de dezembro de 2018 no sistema informatizado de internação do INCA.

Posteriormente, identificaram-se aqueles que atendiam aos seguintes critérios de inclusão: internações com discriminação médica clara de que a criança ou adolescente encontrava-se fora de possibilidades curativas atuais (FPCA), fora de possibilidades terapêuticas atuais (FPTA), em cuidados paliativos (CP) ou cuidados paliativos exclusivos (CPE), considerando que no passado os pacientes eram encaminhados a cuidados paliativos apenas quando se esgotavam as terapêuticas curativas e tratou-se de uma pesquisa retrospectiva, ou seja, apenas internações que apresentavam com clareza que as crianças ou adolescentes não recebiam mais cuidados curativos para a doença.

Excluíram-se da amostra internações pertencentes a pessoas com mais de 18 anos, internações cujos relatos foram inelegíveis ou que não foram localizados por alguma razão, visto que o tempo de recorte da pesquisa foi grande e os prontuários somente passaram a ser eletrônicos no CTIPO no ano de 2017.

Para operacionalização dessa etapa, utilizou-se uma planilha de Excel contendo prontuário, nome e data de entrada e saída de todas as internações de crianças ou adolescentes no CTIPO. Todos os prontuários foram solicitados para verificação dos critérios de inclusão e exclusão do estudo.

A coleta de dados ocorreu entre os meses de novembro de 2018 e fevereiro de 2019. Para a coleta dos dados nas internações incluídas, utilizou-se um instrumento norteador (elaborado pelas autoras), que garantiu a padronização e a sequência lógica na coleta dos dados. 
Para as internações selecionadas, coletaram-se as informações referentes ao diagnóstico oncológico de base, data do diagnóstico, sexo, idade, religião, endereço, data em que a criança e/ou adolescente foi considerado (FPCA, FPTA, CP ou CPE) e a data do óbito quando presente.

$\mathrm{Na}$ análise dos dados, utilizaram-se o programa Statistical Package for the Social Science (SPSS), versão 22.0, e Microsoft Excel 2007.

Para caracterização da amostra e análise descritiva do comportamento das variáveis, os dados foram sintetizados por meio de estatísticas descritivas, gráficos e distribuições de frequências. A variabilidade da distribuição de uma variável quantitativa foi considerada baixa, se $\mathrm{CV}<0,20$; moderada, se $0,20 \leq \mathrm{CV}<0,40$; e alta, se $\mathrm{CV} \geq 0,40$.

$\mathrm{Na}$ análise inferencial de variáveis qualitativas, para verificar a associação entre dois fatores, utilizou-se o Teste qui-quadrado. Quando este se mostrou inconclusivo e foi adequado, usou-se o Teste Exato de Fisher. Na análise Inferencial de Variáveis Quantitativas, dado o pequeno tamanho amostral de um dos subgrupos (menor que 30), a comparação de dois grupos independentes foi feita pelo teste não paramétrico de Mann-Whitney.

A análise de sobrevivência dos pacientes, desde o diagnóstico e início do tratamento paliativo, foi feita pela metodologia de Kaplan-Meie. O tempo de sobrevivência de crianças e adolescentes foi comparado pelo teste de Breslow e teste de Tarone-Ware.

Todas as discussões acerca dos testes de significância foram realizadas considerando o nível de significância máximo de $5 \%(0,05)$.

\section{Resultados}

Identificaram-se 2214 internações, entre os anos de 2008 a 2018, com informações extraídas de 1148 prontuários. Das 2214 internações de crianças e adolescentes, 57 constituíram a amostra do estudo.

Das 57 internações, 37 eram crianças com idade menor que 12 anos $(64,9 \%)$ e $20(35,1 \%)$ eram adolescentes entre 12 e 17 anos. A diferença entre essas proporções é significativa sob o ponto de vista estatístico ( $p$-valor=0,034 do teste Binomial), ou seja, o predomínio de crianças observado na amostra é significativo. A tabela 1 mostra as distribuições de frequências de variáveis referentes ao endereço, sexo, religião, ida-
Tabela 1. Características das crianças/adolescentes internados no CTIPO em CPE, global e por grupo etário crianças e adolescentes

\begin{tabular}{|c|c|c|c|c|}
\hline Variáveis & 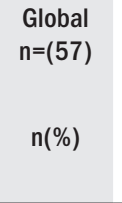 & $\begin{array}{c}\text { Crianças } \\
n=(37) \\
n(\%)\end{array}$ & $\begin{array}{c}\begin{array}{c}\text { Adolescentes } \\
\mathrm{n}=(20)\end{array} \\
\mathrm{n}(\%)\end{array}$ & $\begin{array}{l}p \text {-value do teste } \\
\text { comparando } \\
\text { as distribuições } \\
\text { da variável nos } \\
\text { grupos crianças } \\
\text { e adolescentes }\end{array}$ \\
\hline \multicolumn{5}{|l|}{ Município de origem } \\
\hline Não informado & $5(8,8)$ & $3(8,1)$ & $2(10,0)$ & \multirow{12}{*}{$\begin{array}{l}\text { Teste não } \\
\text { conclusivo }\end{array}$} \\
\hline Rio de Janeiro & $18(31,6)$ & $16(43,2)$ & $2(10,0)$ & \\
\hline $\begin{array}{l}\text { Região } \\
\text { metropolitana }\end{array}$ & $24(42,2)$ & $14(37,8)$ & $10(50,0)$ & \\
\hline $\begin{array}{l}\text { Região do Médio } \\
\text { Paraíba }\end{array}$ & $3(5,3)$ & $2(5,4)$ & $1(5,0)$ & \\
\hline Angra dos Reis & $1(1,8)$ & $0(0,0)$ & $1(5,0)$ & \\
\hline Arraial do Cabo & $1(1,8)$ & $0(0,0)$ & $1(5,0)$ & \\
\hline Campos dos & & & & \\
\hline Goyatacazes & $1(1,8)$ & $0(0,0)$ & $1(5,0)$ & \\
\hline Friburgo & $1(1,8)$ & $0(0,0)$ & $1(5,0)$ & \\
\hline Juiz de Fora & $1(1,8)$ & $1(2,7)$ & $0(0,0)$ & \\
\hline Maceió & $1(1,8)$ & $0(0,0)$ & $1(5,0)$ & \\
\hline São Paulo & $1(1,8)$ & $1(2,7)$ & $0(0,0)$ & \\
\hline \multicolumn{5}{|l|}{ Sexo } \\
\hline Feminino & $33(57,9)$ & $21(56,8)$ & $12(60,0)$ & \multirow[t]{2}{*}{$0,813^{*}$} \\
\hline Masculino & $24(42,1)$ & $16(43,2)$ & $8(40,0)$ & \\
\hline \multicolumn{5}{|l|}{ Religião que segue } \\
\hline Não respondeu & $5(8,8)$ & $3(8,1)$ & $2(10,0)$ & \multirow{6}{*}{$\begin{array}{l}\text { Teste não } \\
\text { conclusivo }\end{array}$} \\
\hline Católica & $20(35,1)$ & $14(37,8)$ & $6(30,0)$ & \\
\hline Cristã & $4(7,0)$ & $2(5,4)$ & $2(10,0)$ & \\
\hline Evangélica & $22(38,6)$ & $13(35,1)$ & $9(45,0)$ & \\
\hline Não tem religião & $4(7,0)$ & $4(10,8)$ & $0(0,0)$ & \\
\hline Outras & $2(3,5)$ & $1(2,7)$ & $1(5,0)$ & \\
\hline \multicolumn{5}{|l|}{ Idade } \\
\hline $1 \mid-4$ & $19(33,3)$ & $19(51,3)$ & $0(0,0)$ & \multirow[t]{5}{*}{$0,000^{* *}$} \\
\hline $4 \mid-8$ & $12(21,1)$ & $12(32,4)$ & $0(0,0)$ & \\
\hline $8 \mid-12$ & $6(10,6)$ & $6(16,2)$ & $0(0,0)$ & \\
\hline $12 \mid-15$ & $9(15,9)$ & $0(0,0)$ & $9(45,0)$ & \\
\hline $15 \mid-18$ & $11(19,3)$ & $0(0,0)$ & $11(55,0)$ & \\
\hline \multicolumn{5}{|l|}{ Diagnóstico } \\
\hline Neuroblatoma & $15(26,3)$ & $15(40,5)$ & $0(0,0)$ & \multirow{15}{*}{$\begin{array}{l}\text { Teste não } \\
\text { conclusivo }\end{array}$} \\
\hline Tumor de SNC & $11(19,3)$ & $5(13,5)$ & $6(30,0)$ & \\
\hline Rabdomiossarcoma & $7(12,3)$ & $2(5,4)$ & $5(25,0)$ & \\
\hline Retinoblatoma & $7(12,3)$ & $7(18,9)$ & $0(0,0)$ & \\
\hline Leucemia & $5(8,8)$ & $3(8,1)$ & $2(10,0)$ & \\
\hline Osteossarcoma & $2(3,5)$ & $0(0,0)$ & $2(10,0)$ & \\
\hline Tumor de Wilms & $2(3,5)$ & $1(2,7)$ & $1(5,0)$ & \\
\hline $\begin{array}{l}\text { Dermatofibrossarcoma } \\
\text { peniano }\end{array}$ & $1(1,8)$ & $0(0,0)$ & $1(5,0)$ & \\
\hline Linfoma & $1(1,8)$ & $0(0,0)$ & $1(5,0)$ & \\
\hline PNET abdominal & $1(1,8)$ & $1(2,7)$ & $0(0,0)$ & \\
\hline PNET de coluna & $1(1,8)$ & $1(2,7)$ & $0(0,0)$ & \\
\hline PNET ilíaco & $1(1,8)$ & $1(2,7)$ & $0(0,0)$ & \\
\hline $\begin{array}{l}\text { Sarcoma de Membro } \\
\text { inferior }\end{array}$ & $1(1,8)$ & $0(0,0)$ & $1(5,0)$ & \\
\hline $\begin{array}{l}\text { Tumor desmoplásico } \\
\text { abdominal }\end{array}$ & $1(1,8)$ & $0(0,0)$ & $1(5,0)$ & \\
\hline $\begin{array}{l}\text { Tumor Rabdoide } \\
\text { renal }\end{array}$ & $1(1,8)$ & $1(2,7)$ & $0(0,0)$ & \\
\hline
\end{tabular}


de e doença oncológica de base, que caracterizam as crianças/adolescentes internados no CTIPO em CPE, global e por grupo etário.

\section{Acompanhamento da família}

Das 57 internações, 1 adolescente $(1,8 \%)$ não tinha acompanhamento da família. A tabela 2 traz a distribuição de frequências do grupo familiar que acompanhou as crianças/adolescentes internados no CTIPO em CPE.

Tabela 2. Distribuição das frequências dos familiares/grupos familiares acompanhantes das crianças/adolescentes em cuidados paliativos exclusivos no Centro de Terapia Intensiva Pediátrica

\begin{tabular}{l|c|c|c}
\hline Acompanhante & $\begin{array}{c}\text { Global } \\
\mathbf{n}=(57) \\
\mathbf{n}(\%)\end{array}$ & $\begin{array}{c}\text { Crianças } \\
\mathbf{n}=(37) \\
\mathbf{n}(\%)\end{array}$ & $\begin{array}{c}\text { Adolescentes } \\
\mathbf{n}=(20) \\
\mathbf{n}(\%)\end{array}$ \\
\hline Mãe & $31(54,4)$ & $20(54,1)$ & $11(55,0)$ \\
Mãe e pai & $11(19,3)$ & $4(10,8)$ & $7(35,0)$ \\
Pai, mãe e tia & $3(5,3)$ & $3(8,1)$ & $0(0,0)$ \\
Mãe e avó & $2(3,5)$ & $2(5,4)$ & $0(0,0)$ \\
Mãe e tia & $2(3,5)$ & $1(2,7)$ & $1(5,0)$ \\
Avó & $2(3,5)$ & $2(5,4)$ & $0(0,0)$ \\
Pai, mãe e avó & $1(1,8)$ & $1(2,7)$ & $0(0,0)$ \\
Mãe, pai e tia & $1(1,8)$ & $1(2,7)$ & $0(0,0)$ \\
Avó e tia & $1(1,8)$ & $1(2,7)$ & $0(0,0)$ \\
Pai & $1(1,8)$ & $1(2,7)$ & $0(0,0)$ \\
Tia & $1(1,8)$ & $1(2,7)$ & $0(0,0)$ \\
Nenhum acompanhante & $1(1,8)$ & $0(0,0)$ & $1(5,0)$ \\
\hline
\end{tabular}

\section{Sobrevivência e óbito}

Das 57 internações de crianças/adolescentes em CPE, $53(93,0 \%)$ foram a óbito no período observado (até 31/01/2018). Pelo método de Kaplan-Meier, estimou-se que, no global, o tempo médio de sobrevivência desde o início do CPE foi de aproximadamente 7,6 dias com intervalo de confiança de 1,$7 ; 13,4$. O tempo mediano de sobrevivência a partir do início dos CPE foi de 0,6 dia com intervalo de confiança de 0,$3 ; 0,9$, como pode ser visto na tabela 3 .
Outro dado coletado nos prontuários mostrou que, das 57 internações de crianças e adolescentes, 12 (21\%) fecharam CPE antes de entrarem no CTIPO e 45 (78,9\%) fecharam CPE no CTIPO. Para os que fecharam o diagnóstico de CPE antes de entrarem no CTI$\mathrm{PO}$, o tempo entre o diagnóstico e a entrada do CTIPO variou de 2 a 433 dias $(\mathrm{CV}=1,3)$, mediana de 22 dias, média de 108,2 e desvio padrão de 145,8 dias. Para os que fecharam o diagnóstico de CPE no CTIPO, o tempo entre a entrada do CTIPO e o diagnóstico variou de 0 a 168 dias $(\mathrm{CV}=1,4)$, mediana de 11 dias, média de 22,4 dias e desvio padrão de 31,6.

\section{Discussão}

Os dados desta pesquisa trazem 57 internações de crianças/adolescentes de 0-17 anos em CPE que estiveram internados no CTIPO entre 2008 e 2018. Essas internações eram em sua maioria de crianças entre 1 e 11 anos $(64,9 \%)$ e em menor proporção adolescentes entre 12 e 17 anos (35,1\%), sendo a idade média entre as crianças de 4,2. Esses dados corroboram com estudos de incidência de câncer geral infantojuvenil, que mostram as faixas etárias mais precoces (0-4 anos) como mais propensas ao desenvolvimento de neoplasias. ${ }^{(5)}$

Quanto ao aspecto demográfico e entendendo o INCA como sendo uma instituição federal de âmbito nacional e internacional, ${ }^{(6)}$ observou-se que $31,6 \%$ das crianças e adolescentes da amostra eram residentes na cidade do Rio de Janeiro e 54,3\% de municípios do estado do Rio de Janeiro, sendo apenas 5,2\% de crianças e adolescentes provenientes de outros estados.

Esses resultados ressaltam a política da Rede de Atenção Oncológica do SUS. Essa rede possui Unidades de Assistência de Alta Complexidade em Oncologia (UNACON) e Centros de Assistência de Alta Complexidade em Oncologia (CACON).(7) Há pelo menos um hospital especializado em tratamento de câncer em cada estado brasileiro, ${ }^{(7)}$ favorecendo a descentra-

Tabela 3. Estimativas da média e mediana do tempo de sobrevivência (em dias) desde o início do CPE das crianças/adolescentes internadas no CTIPO, global e por grupo etário. Rio de Janeiro, RJ (2019)

\begin{tabular}{lcccccccc}
\hline Grupo etário & Estimativa & Erro padrão & \multicolumn{2}{c}{$\begin{array}{c}\text { Média } \\
\text { Intervalo de confiança ao nível de (95\%) } \\
\text { Limite inferior }\end{array}$} & Limite superior & Estimativa & Erro padrão & $\begin{array}{c}\text { Mediana } \\
\text { Intervalo de confiança ao nível de (95\%) } \\
\text { Limite inferior } \\
\text { Limite superior }\end{array}$ \\
\hline Global & 7,6 & 3,0 & 1,7 & 13,4 & 0,6 & 0,2 & 0,3 & 0,9 \\
Crianças & 5,4 & 2,1 & 1,4 & 9,5 & 0,6 & 0,1 & 0,3 & 0,8 \\
Adolescentes & 6,9 & 5,2 & 0,0 & 17,0 & 0,6 & 0,3 & 0,0 & 1,3 \\
\hline
\end{tabular}


lização no atendimento e propiciando às crianças/adolescentes a realização do tratamento próximo as suas residências.

A religião e a espiritualidade são fontes de conforto e esperança para crianças/adolescentes e cuidadores diante de um momento desafiador. ${ }^{(8)}$ Como a pesquisa se deu por meio de análise documental, não foi possível inferir sobre a espiritualidade, pois esta é vista como uma orientação de cunho filosófico que produz comportamentos e sentimentos como esperança, amor e fé. ${ }^{(8)}$ Já a religiosidade está atrelada à fé, a busca por um ser superior para ajudá-las no enfrentamento das situações difíceis, mas sempre acreditando na capacidade de recuperação e cura da criança/adolescente. ${ }^{(8)}$

Os dados de religiosidade mostram que as religiões cristãs (evangélica e católica) faziam parte das crenças de 73,6\% das crianças/adolescentes e suas famílias. Esse resultado justifica-se pelo fato de o Brasil ser considerado um país cristão, considerando que se autointitulam cristãos mais de $80 \%$ dos brasileiros filiados a uma religião. ${ }^{(9)}$

Em relação ao diagnóstico do câncer, obteve-se como resultado no percentual geral o neuroblastoma como mais incidente $(26,3 \%)$, seguido dos tumores do Sistema Nervoso Central (SNC) (19,3\%), e o rabdomiossarcoma e retinoblastoma, ambos com 12,3\%, como segundo e terceiros lugares, respectivamente.

Analisando o perfil de gravidade dos cânceres mais incidentes na amostra, tem-se o neuroblastoma que é um tipo de câncer com prognóstico ruim em crianças maiores de 1 ano, além disso, 46\% delas apresentam metástases ao diagnóstico. ${ }^{(10)}$ Os Tumores do SNC apresentam uma grande variedade histológica, representando o segundo grupo de diagnóstico mais comum ( $17 \%$ a $25 \%$ ) das neoplasias na infância, além de ser o tumor sólido mais frequente e a principal causa de mortalidade por câncer em jovens, tendo maior probabilidade de serem metastáticos ao diagnóstico. ${ }^{(11)}$

O rabdomiossarcoma é o tumor de partes moles mais comum na infância, está relacionado a mau prognóstico por ser agressivo. Quanto às crianças, 25\% apresentam metástases ao diagnóstico e os principais locais de acometimento são pulmões, medula óssea, ossos e linfonodos. ${ }^{(12)}$

Identificou-se que 12 crianças/adolescentes (21\%) fecharam CPE antes de entrarem no CTIPO e 45 $(78,9 \%)$ fecharam CPE depois que entraram no CTPO.
Quanto às 12 crianças/adolescentes que fecharam CPE antes de entrarem no CTIPO, podem ter entrado por diversas razões (que não foram comprovadas no estudo). Entretanto, das 45 crianças/adolescentes que fecharam CPE após a internação no CTIPO, observou-se que esse diagnóstico veio em um intervalo de poucas horas ou dias após a internação e todos faleceram em um intervalo médio de 7,6 dias dentro da unidade de tratamentos intensivos.

Estudo realizado no Hospital Universitário do Rio Grande do Norte avaliou os CP pediátricos e trouxe como resultado que os profissionais de saúde possuem dificuldades em aceitar a finitude e acreditam que sempre há algo mais a se fazer pelas crianças/adolescente, culminando no início tardio dos $\mathrm{CP} .{ }^{(13)}$

Com o progresso da tecnologia médica, tornou-se possível prolongar a vida de pacientes portadores de doenças irreversíveis e de prognóstico reservado, muitas vezes esse prolongamento ocorre apesar de não proporcionar qualidade de vida e aumentar o sofrimento de crianças/adolescentes e seus familiares. ${ }^{(14)}$

Um dos fatores que levam à obstinação terapêutica ou prolongamento da vida em crianças e adolescentes com cânceres incuráveis advém da dificuldade emocional das equipes de saúde em conversar abertamente sobre prognóstico e objetivos dos cuidados com crianças/ adolescentes e suas famílias nos casos em que o risco de morte é eminente por confundir CPE com a ideia de se "perder a esperança", desistir e o que é pior admitir sua impotência em relação ao tratamento curativo. ${ }^{(14)}$

Consequentemente, é um equívoco comum iniciar o CPE apenas no final da vida, é frequente que crianças e adolescentes em estágio avançado do câncer sejam internados em estado grave e com risco de morte no CTIPO, sem terem recebido nenhuma abordagem paliativa anterior, ficando a cargo dos profissionais do CTIPO iniciarem essa conversa com a família. ${ }^{(15)}$

Faz-se necessário um aporte humanístico, competências e habilidades relativas ao relacionamento interpessoal, para que se tenham condições de compreender e ajudar a criança/adolescente e sua família no contexto do processo de morte e morrer tão difícil e temido por todos. ${ }^{(14)}$ Assim, quando a ciência dos CP é atrelada à arte de cuidar, envolvendo a sensibilidade, atitudes de carinho, apoio psicológico, psicossocial e espiritual, crianças/adolescentes e famílias sentem-se mais seguros em aceitar a morte como natural e evitar 
medidas invasivas, como geralmente são oferecidas no CTIPO.

Concernente ao direto das crianças e adolescentes garantido pelo Estatuto da Criança e do Adolescente (ECA) ${ }^{(16)}$ de acompanhamento durante as 24 horas de internação hospitalar, os dados mostram que apenas $1(1,8 \%)$ adolescente permaneceu sem acompanhante.

As mães permaneceram acompanhando sozinhas em $54,4 \%$ das vezes. Assim, quando somamos as vezes em que estiveram sozinhas com as vezes em que ficaram com outros familiares, elas permaneceram no hospital acompanhando seus filhos $89,4 \%$ do tempo.

As mães de crianças e adolescentes com câncer são na maioria das vezes a principal referência de dedicação, presença e zelo. As mães lidam com o desafio de acompanhar todo o processo do diagnóstico, tratamento, internações, reinternações e as notícias difíceis ao lado do filho. Vivenciam uma experiência de grande estresse físico e emocional por diferentes situações, como o próprio sofrimento e o medo constante da morte. ${ }^{(17)}$

Entretanto, é uma luta que a mãe enfrenta muitas vezes sem sentir-se preparada. ${ }^{(18)}$ Os profissionais devem estar atentos a situações de desgaste físico e emocional por parte dessas mães. É preciso atuar em prol do resgaste da rede de apoio familiar, procurar entender os motivos do pouco rodízio entre os familiares, promovendo $\mathrm{CP}$ nos aspectos psicossociais.

Quanto aos achados da pesquisa que se refere à incidência de mortalidade por câncer em crianças e adolescentes, obteve-se um percentual de 93\% de óbitos em relação à amostra total de 57 crianças/adolescentes até a data final estipulada para coleta de dados nos prontuários (31/12/2018), o que se justifica pelo perfil de crianças e adolescentes incluídos na amostra, todos com impossibilidade de tratamento modificador da doença.

O câncer geralmente é considerado mais agressivo na criança do que no adulto, porém esta responde melhor ao tratamento devido ao seu processo acelerado de divisão celular. ${ }^{(1,11)}$ Contudo, o atraso no diagnóstico e consequentemente no início do tratamento favorece o constante aumento de crianças/adolescentes com cânceres metastáticos ao diagnóstico, sendo considerados a principal causa de morte por doenças nessa faixa etária no Brasil. ${ }^{(19)}$

\section{Conclusão}

O câncer infantojuvenil é um problema de saúde pública, que embora tenha um potencial de cura elevado em comparação com a população adulta, são muitos os casos de detecção tardia e/ou de tumores agressivos que evoluem sem resposta ao tratamento curativo. Em vista disso, estratégias em prol da capacitação dos profissionais da atenção primária à saúde para o reconhecimento dos sinais e sintomas do câncer infantojuvenil e articulações intersetoriais para o encaminhamento e início precoce de tratamento dos casos confirmados são necessárias para a redução da mortalidade por câncer nessa faixa etária. Igual atenção deve ser dada à formação dos profissionais na área da saúde a respeito da terminalidade e CP para que crianças e adolescentes com câncer fora de possibilidade de cura possam desfrutar com qualidade os últimos momentos da sua vida. Assim, este estudo possibilitou o conhecimento do perfil clínico e demográfico dessas crianças/adolescente com impossibilidade de tratamento modificador da doença de um hospital de referência no tratamento de câncer no Rio de Janeiro a fim de que com a utilização desses dados possam ser oferecidos CP de forma precoce, bem como valorizar as redes de apoio, valores, desejos e biografias de cada criança/adolescente e sua família. Sabemos que o CP ainda que definido e propagado mundialmente pela OMS no final dos anos 90, no Brasil uma política que de fato o incorporou no Sistema Único de Saúde como parte integrante das redes assistenciais surgiu em outubro de 2018. Isso gera a reflexão sobre a imaturidade dos enfermeiros no processo de pensamento que leva à decisão clínica acerca da promoção da qualidade de vida, posto que a ausência de política pública sobre o $\mathrm{CP}$ propiciou a escassez de ensino e discussões nas universidades e educação permanente nas unidades de saúde. Diante disso, a pesquisa colabora com a promoção da segurança das crianças e adolescentes hospitalizadas no CTIPO em $\mathrm{CP}$, já que o conhecimento pode ser considerado um importante fator para identificar, prevenir e minimizar os efeitos do sofrimento evitável proveniente da prática profissional, uma vez que busca organizar uma prática baseada em evidência, fundamentada cientificamente, respaldada legalmente e documentada, contribuindo, assim, para a segurança. Similarmente, a pesquisa contribui com a ampliação 
dos conhecimentos em CP, além de fortalecer a produção de conhecimentos em enfermagem no âmbito da saúde da criança/adolescente com câncer perante a lacuna do conhecimento sobre essa problemática. Nesse contexto, o enfermeiro pediatra tem um importante papel na assistência à criança/adolescente e sua família, desde o diagnóstico do câncer até o desfecho de cura ou impossibilidade de cura e morte, ofertando o CP de modo oportuno em associação com o curativo para melhor qualidade de vida possível ao longo do tratamento, independente do prognóstico da doença.

\section{Agradecimentos}

Agradecemos ao Conselho Federal de Enfermagem COFEN e a Coordenação de Aperfeiçoamento de Pessoal de Nível Superior - CAPES que estão financiando este estudo, no que diz respeito a sua divulgação e publicação.

\section{Contribuições}

Silva e Sousa ADR, Silva LF, Cavalcanti ACD, Silva MM, Góes FGB, Moraes JRMM e Anjos C contribuíram com a concepção do projeto, análise e interpretação dos dados, redação do artigo, revisão crítica relevante do conteúdo intelectual e aprovação final da versão a ser publicada.

\section{Referências}

1. Instituto Nacional do Câncer Jose Alencar Gomes da Silva (INCA). ABC do Câncer: Abordagens básicas para 0 controle do câncer [Internet]. Rio de Janeiro: INCA; 2019. [citado 2019 Nov 15]. Disponível em: https://www.inca.gov.br/sites/ufu.sti.inca.local/ files//media/document//livro-abc-4-edicao.pdf
2. Instituto Nacional do Câncer Jose Alencar Gomes da Silva (INCA). Estimativas/2018: Incidência de câncer no Brasil [Internet]. Rio de Janeiro: INCA; 2018. [citado 2019 Nov 15]. Disponível em: http://www1.inca.gov.br/inca/Arquivos/estimativa-2018. pdf

3. Iglesias SB, Zollner AC, Constantino CF. Cuidados paliativos pediátricos. Resid Pediátr. 2016;6(supl1):46-54.

4. World Health Organization (WHO). Palliative Care. Geneva: WHO; 2017 [cited 2019 Aug 18]. Available from: http://www.who.int/mediacentre/factsheets/fs402/en/.

5. Monteiro NM, Fernandes FL, Soares IA, Hyodo LT. Perfil Clínico e Epidemiológico dos Pacientes de um Serviço de Oncologia Pediátrica de um Hospital do Leste de Minas Gerais. Rev Med Minas Gerais. 2018;28(e-1961):1-7.

6. Instituto Nacional do Câncer Jose Alencar Gomes da Silva (INCA). Sobre o INCA. [Internet]. Rio de Janeiro: INCA; 2020. [citado 2020 Jun 102]. Disponivel em: https://www.inca.gov. br/institucional

7. Portal da Educação Tecnologia Educacional LTDA. Rede de Atenção Oncológica no Brasil. São Paulo: Portal Educação; 2019.

8. Alves DA, Silva LG, Delmondes GA, Lemos IC, Kerntopf MR, Albuquerque GA. Cuidador de criança com câncer: religiosidade e espiritualidade como mecanismos de enfrentamento. Rev Cuid. 2016;7(2):1318-24.

9. Alves JE, Cavenaghi S, Barros LP, Carvalho AA. Distribuição espacial da transição religiosa no Brasil. Tempo Soc. 2017;29(2):215-42.

10. Lucena JN, Alves MT, Abib SC, Souza GO, Neves RP, Coran EM. Aspectos clínicos, epidemiológicos e sobrevida de crianças com neuroblastoma: 21 anos de experiências do Instituto de Oncologia Pediátrica, São Paulo. Rev Paul Pediatr. 2018;36(3):254-60.

11. Oliveira AT, Sousa MN, Maia PC, Bezerra AL, Moura BC, Santos EV, et al. Perfil epidemiológico do câncer infantil na Paraíba. REAS. 2019;11(16):e1568.

12. Cunha NC, Suzuki Al, Lima FF, Fernandez PV, Faria PA, Fernandez TS, et al. Alterações Citogenético-Moleculares no Gene F0X01 em uma Criança com Rabdomiossarcoma Alveolar: Relato de Caso. Rev Bras Cancerol. 2018;64(3):415-9.

13. Ferreira MG, Iglesias SB. Cuidados paliativos pediátricos, terminalidade e espiritualidade: Estamos preparados? Resid Pediátr. 2019;9(1):53-7.

14. Barros KG, Gonçalves JR. Aspectos psicológicos que envolvem os cuidados paliativos pediátricos. Rev. JRG Estud. Acad. 2019;2(5):156-65.

15. Baptista SC, Picanço CM. Cuidados paliativos em unidade de atendimento crítico: saberes de uma equipe multiprofissional. Enferm Bras. 2019;(1895):612-24.

16. Brasil. Ministério da Justiça. Lei 13.257 , de 8 de Março de 2016. Dispõe sobre a proteção integral à criança e ao adolescente. Estatuto da Criança e do Adolescente. [Internet]. Brasília (DF): Ministério da Justiça; 2016. [citado 2020 Out 3]. Disponivel em: https:// www.in.gov.br/materia/-/asset_publisher/Kujrw0TZC2Mb/content/id/21172863/do12016-03-09-lei-no-13-257-de-8-de-marco-de-2016-21172701

17. Vidotto PC, Ferrari RA, Tacla MT, Facio BC. Experiência materna no itinerário diagnóstico do câncer infantil. Rev Enferm UFPE on line. 2017;11(4):1565-73.

18. Costa MA, Agra J, Santos NC, Oliveira CD, Freire ME, Costa MM. Experiências de mães de crianças com câncer em cuidados paliativos. Rev Enferm UFPE on line. 2018;12(5):135564.

19. Ries PK, Costenaro RG. Fatores relacionados ao diagnóstico tardia das neoplasias na infância e adolescência. Discip Sci Sér Ciênc Biol Saúde. 2017;18(1):111-21. 